\title{
Metastatic Prostate Cancer Initially Presenting as
}

\section{Supraclavicular Lymphadenopathy}

\section{Sonam $S^{*}$ and Shipra $S$}

Department of Pathology, Vardhman Mahavir Medical College \& Safdarjung Hospital, New Delhi, India

*Corresponding author: Sonam Sharma, Department of Pathology, Vardhman Mahavir Medical College \& Safdarjung Hospital, New Delhi, India, Tel: +919999841393;

\section{Case Report \\ Volume 2 Issue 1}

Received Date: January 25, 2017

Published Date: March 09, 2017

DOI: $10.23880 /$ ooaj-16000148

drsonamsharma@gmail.com

\section{Abstract}

Prostate cancer is one of the most common neoplasm occurring in men worldwide. It can present with diverse clinicopathological features that can cause a diagnostic conundrum. We report one such rare and an unusual manifestation of this cancer in a 40-year-old male who initially presented with supraclavicular lymph node enlargement. There are very few cases in the literature which have described supraclavicular lymphadenopathy as the initial presentation of metastatic prostate cancer in men younger than 45 years of age. These patients rarely undergo digital rectal examination or serum prostate-specific antigen level measurement as part of their initial investigations. A high index of suspicion is necessary to make the diagnosis of prostate cancer in such atypical cases.

Keywords: Prostate cancer; Metastasis; Young age group; Supraclavicular lymphadenopathy

Abbreviations: PSA: Prostate-Specific Antigen; FNAC: Fine Needle Aspiration Cytology; AMCAR: AlphaMethylacyl Coenzyme A Racemase; LCA: Leucocyte Common Antigen; TTF1: Thyroid Transcription Factor 1; WT-1: Wilm's tumor suppressor gene 1 protein; PLAP: Placental Alkaline Phosphatase; ER: Estrogen Receptor; PR: Progesterone receptor; CK: Cytokeratin; CT: Computed Tomography; ASH: Atypical Adenomatous Hyperplasia

\section{Introduction}

Carcinoma of the prostate is the second leading cause of cancer-related deaths in males in the world with 905,330 and 1.1 million cases and 258,400 and 307,000 deaths in 2008 and 2011, respectively. Almost 70\% cases were from developed countries. The quantum of carcinoma prostate burden worldwide is expected to increase to 1.7 million new cases and 499,000 new deaths by 2030 because of population explosion and aging [1,2]. Peak incidence occurs between the ages of 70 to 74 years, less than $0.1 \%$ of all patients with prostate cancer are younger than 50 years of age [3]. It can have variety of presentations, including asymptomatic with normal or raised prostate-specific antigen (PSA) levels, local invasion and regional lymph node involvement or metastasis with systemic symptoms. The most common sites for its metastasis are regional lymph nodes (the obturator, internal and external iliac nodes, followed by the presacral and para-aortic node), bones, lungs, liver, brain and the epidural space [4]. Distant metastasis are relatively rare at diagnosis, and include supraclavicular, mediastinal, pulmonary and retroperitoneal nodes $[5,6]$. This report describes a case of prostate cancer metastatic to the left supraclavicular lymphnode in a 40-year-old male who was initially clinically misdiagnosed as a case of lymphoma. 


\section{Otolaryngology Open Access Journal}

\section{Case Report}

A 40-year-old male presented to the surgical outpatient department with the chief complaints of left supraclavicular mass since last 2 months. He also gave history of anorexia and weight loss. He denied any other subjective complaints, like difficulty in swallowing, breathing, urinary symptoms or bone pains. His past history, medical and family history were noncontributory. He had a 10-year history of smoking and drinking alcohol. On examination, the mass measured 3 $\mathrm{cm} \times 3 \mathrm{~cm}$ in size and was firm in consistency. It was nontender and fixed to the underlying tissues. The overlying skin was normal. Further physical examination revealed palpable nodes (ranging in size from $0.3 \mathrm{~cm}$ to $1.5 \mathrm{~cm}$ ) in the left axilla. Rest of the physical examination, the inguinal region, external genitalia, scrotum and testes were unremarkable. A clinical diagnosis of lymphoma was made. Routine haematological and biochemical investigations were within normal limits expect for an elevated creatinine $(4.8 \mathrm{mg} / \mathrm{dl})$, blood urea nitrogen $(102$ $\mathrm{mg} / \mathrm{dl})$ and PSA levels $(40 \mathrm{ng} / \mathrm{ml})$. A fine needle aspiration cytology (FNAC) of the supraclavicular mass was performed. On FNAC, the smears were highly cellular and showed presence of atypical cells with decreased cell cohesion and variable isolated cells. Micro glandular patterns were evident. The cells had indistinct cell membranes with high nuclear to cytoplasmic ratio and significant nuclear pleomorphism with prominent nucleoli in some. In the background of these cells, many reactive lymphoid cells in various stages of maturation were seen. Based on these findings, a possibility of metastatic carcinoma was suggested.

An excisional biopsy of the left supraclavicular mass was performed. Microscopic sections of this mass revealed a lymph node which was diffusely infiltrated by a tumor. The tumor cells were arranged in tubules and glandular structures. The individual tumor cell showed the presence of round to oval vesicular nuclei with prominent nucleoli and abundant amphophylic cytoplasm (Figure 1). Atypical mitosis was also seen. A panel of immunohistochemical markers were put. The tumor cells were positive for pan- cytokeratin (CK), Muc-1, PSA (Figure 2a) and alpha-methylacyl coenzyme A racemase (AMCAR) (Figure 2b) while they were negative for leucocyte common antigen (LCA), thyroid transcription factor 1 (TTF1), CK7, CK20, p-53, Bcl-2, CD117, p63, CD10, Wilm's tumor suppressor gene 1 protein (WT-1), placental alkaline phosphatase (PLAP), estrogen receptor (ER), progesterone receptor (PR), synaptophysin, chromogranin, CD34, vimentin, desmin and S100.
Considering the morphological and immunohistochemical findings, a diagnosis of prostatic adenocarcinoma metastatic to the left supraclavicular lymphnode was made. Following this, a detailed clinical and radiological workup was advised to confirm the primary.

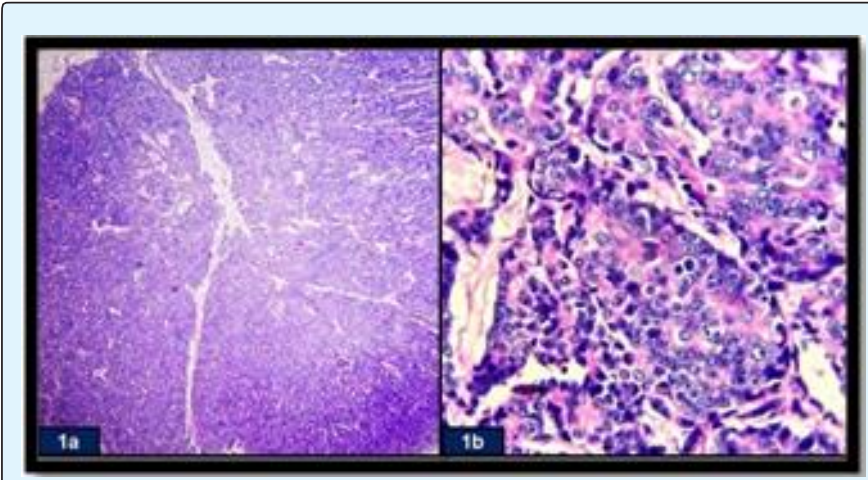

Figure 1: a) Excision biopsy of the left supraclavicular mass showing metastatic adenocarcinoma of undetermined origin ( $\mathrm{H} \& \mathrm{E}, \mathrm{x} 10)$.

b) Tumor composed of malignant cells with vesicular nuclei and prominent nucleoli (H \& E, x40).

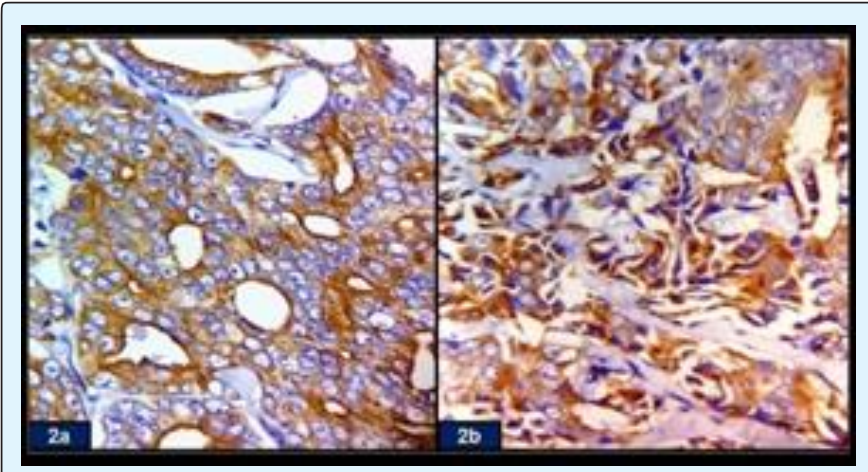

Figure 2: a. Immunohistochemical staining of the neoplastic cells, exhibiting strong positivity for PSA (IHC, $\mathrm{x} 40$ ).

b. AMACR positivity of the tumor cells (IHC, $\mathrm{x} 40$ ).

He underwent a computed tomography (CT) scan of the neck, chest and abdomen. The CT scan demonstrated adenopathy within the supraclavicular, left axillary and pelvic region. A prostate mass was evident. There was bilateral hydronephrosis with no evidence of any bone metastasis. The patient was referred to urology department for further evaluation. Digital rectal examination was done and it revealed a stony hard, fixed, and enlarged prostate. Transrectal ultrasonography guided prostate biopsy revealed adenocarcinoma with a Gleason score of $5+4=9$. A Tc- $99 \mathrm{~m}$ whole body scan revealed no skeletal metastasis. Based on these clinicoradiological and pathological findings, a final 


\section{Otolaryngology Open Access Journal}

diagnosis of clinically occult prostatic adenocarcinoma metastatic to the left supraclavicular lymph node was established. The patient was initiated with androgen deprivation therapy with bicalutamide and leuprorelin acetate. Six months later, his enlarged left supraclavicular lymph node had disappeared and serum PSA had decreased to $2.91 \mathrm{ng} / \mathrm{mL}$.

\section{Discussion}

The most common cause of cervical lymph node enlargement in children and young adults is usually related to an inflammatory condition or lymphoma. On the other hand, in the elderly age group, lymphoma or metastatic cancers, constitute the major bulk. Most cancers that present as cervical lymphadenopathy are derived from head and neck malignancies involving the mucosal surfaces of the aerodigestive tract, with occasional metastasis from lung, breast, and kidney [7,8]. The incidence of involvement of cervical lymph nodes in patients with prostate cancer has been reported as $0.4 \%$ or less; with only few cases occurring in men younger than 45 years of age $[3,5,7,9]$.

Various hypotheses have been documented in the literature regarding the head and neck metastasis from prostate cancer. First is the hematogenous spread via the vertebral venous system, or Batson's plexus [10] while the other is the lymphatic spread via the lymphatics in the prostate that drain obturator-hypogastric and presacral nodes and from these to the iliac, paraaortic, cisterna chyli, and thoracic duct. Finally, the lymphatic drainage enters the systemic blood circulation via the left subclavian vein. Some authors have also postulated that the tumor cells can lodge in the left cervical nodes by retrograde spread due to the proximity of these nodes with the point-of-entry of the thoracic duct into the left subclavian vein [6]. It has also been described by some researchers that this carcinoma has a predilection to metastasize to the left cervical region lymph nodes and the involvement of right side cervical lymph nodes is extremely uncommon [6-8,11]. The present case was a 40-year-old male who presented with a left supraclavicular mass as the first clinical evidence of prostate cancer. The authors believe that supraclavicular lymph node metastasis in this patient was due to lymphatic spread, as the pattern of lymph node invasion in the neck was limited to the left side. There were many confounding factors for the misdiagnosis in this patient clinically like the patient's age and the absence of urinary complaints which lead to a diagnosis of lymphoma. However, it was the cytology which aided in the differential diagnosis initially and subsequently, it was histopathology along with various immunohistochemical markers which helped in clinching the definite diagnosis.

Despite advances in screening and multimodal management of this disease, overall survival of patients with prostate cancer remains poor. This is owing to variety of benign mimickers of prostate carcinoma such as benign hyperplasia, prostatitis, atrophy, adenosis, atypical adenomatous hyperplasia (AAH) and nephrogenic adenoma, which makes its diagnosis challenging $[12,13]$. Therefore, many pathological parameters are required for its proper assessment. Among them, serum PSA level is a specific marker of prostatic tissue that permits the definition of the prostatic origin of a metastatic adenocarcinoma. However, high levels of PSA may also be observed in benign prostatic hyperplasia and may be normal in poorly differentiated metastatic prostate carcinoma. Epstein \& Eggleston [14] showed that prostate tumours with normal or slightly increased PSA are more aggressive than other types. Normal levels of serum PSA and/or free/total PSA fractions are not sufficient to exclude the diagnosis of prostate cancer. Jones \& Anthony [6] observed an increase in PSA in only 5 (45.5\%) of the 11 cases reported with prostate cancer and cervical lymphadenopathy. The treatment regarding an isolated neck metastasis still remains controversial. While neck dissection has an established role in the treatment of some of the loco-regional malignancies that spread to the cervical lymph nodes, but so far there is lack of experience with prostate cancer metastasis to this site. Some authors suggest that the surgical intervention in such cases is of little utility while others have documented that the supraclavicular lymph node metastasis should be treated as a systemic disease and should be primarily treated with androgen ablation therapy which includes orchiectomy, antiandrogens, adrenal enzyme synthesis inhibitors and gonadotrophin-releasing hormone analogues. Chemotherapy is used in patients with advanced disease after hormone therapy/ androgen suppression therapy has failed [15]. A potential role for radiation lies only in the palliation of local symptoms relating to venous obstruction and/or neural compression. However, despite these speculations, there is lack of knowledge about the early detection or management of prostate cancer in young patients (aged $<55$ years), due to the severe lack of randomized controlled trials regarding this matter. Most authors believe these cancers represent a distinct phenotype in prostate cancer, etiologically, and clinically, and therefore clear guidelines are needed for appropriate management of these patients $[16,17]$. The present case was treated with hormone therapy, to which he responded favourably. 


\section{Otolaryngology Open Access Journal}

\section{Conclusion}

Prostate cancer should always be considered among the differential diagnosis of men presenting with supraclavicular or any cervical lymphadenopathy, irrespective of the age and the absence of lower urinary tract symptoms. A thorough physical examination including digital rectal examination, measurement of serum prostate specific antigen and subsequently cytology and histopathology along with immunohistochemical stains play an important role in establishing the diagnosis of the prostate cancer in such an unusual clinical setting.

\section{References}

1. Perin NN (2001) Global variation in cancer incidence and mortality. Curr Sci 81(5): 465-474.

2. Mannan R, Bhasin TS, Manjari M, Singh G, Bhatia PK, Sharma $S$ et al. (2016) Immunohistochemical expression of Ets-related gene-transcriptional factor in adenocarcinoma prostate and its correlation with Gleason score. Indian J Pathol Microbiol 59(4): 489495.

3. Lin YY, Lin DS, Kang BH, Lin YS (2011) Neck mass as the first presentation of metastatic prostatic adenocarcinoma. J Chin Med Assoc 74(12): 570-573.

4. Epstein JI (2007) Pathology of prostatic neoplasia. In: Wein AJ, et al. (Eds.), Campbell-Walsh Urology. (9 edn), Philadelphia: W.B. Saunders pp. 2874-2875.

5. Hematpour K, Bennett CJ, Rogers D, Head CS (2006) Supraclavicular lymph node: incidence of unsuspected metastatic prostate cancer. Eur Arch Otorhinolaryngol 263(9): 872-874.

6. Jones H, Anthony PP (1992) Metastatic prostatic carcinoma presenting as left-sided cervical lymphadenopahty: a series of 11 cases. Histopathalogy 21(2): 149-154.

7. Sepúlveda L, Gorgal T, Pires V, Rodrigues F (2015) Prostate Cancer Metastatic to the Cervical Lymph Nodes. Case Reports in Urology 2015: 4.

8. Carleton J, van der Riet P, Dahm P (2005) Metastatic prostate cancer presenting as an asymptomatic neck mass. Prostate Cancer Prostatic Dis 8(3): 293-295.
9. Flocks RH, Boatman DL (1973) Incidence of head and neck metastases from genito-urinary neoplasms. Laryngoscope 83(9): 1527-1539.

10. Batson OV (1995) The function of the vertebral veins and their role in the spread of metastases. Clin Orthop Relat Res 312: 4-9.

11. Ozgur A, Ilker Y, Turkeri LN (2003) Cervical lymph node enlargement on the right side as the initial manifestation of metastatic prostate cancer. Arch Esp Urol 56(7): 859-861.

12. Kaur H, Paul M, Manjari M, Sharma S, Bhasin T, et al. (2016) Ki-67 and p53 Immunohistochemical Expression in Prostate Carcinoma: An Experience from a Tertiary Care Centre of North India. Ann Pathol Lab Med 3(6): 509-516.

13. Srigley JR (2004) Benign mimickers of prostatic adenocarcinoma. Mod Pathol 17(3): 328-348.

14. Epstein JI, Eggleston JC (1984) Immunohistochemical localization of prostate-specific acid phosphatase and prostate-specific antigen in stage A2 adenocarcinoma of the prostate: prognostic implications. Hum Pathol 15(9): 853-859.

15. Moura FM, Garcia LT, Castro LPF, Ferrari TCA (2006) Prostate adenocarcinoma manifesting as generalized lymphadenopathy. Urol Oncol Semin Orig Investig 24(3): 216-219.

16. Chitale SV, Harry L, Gaches CGC, Ball RY (2001) Presentation of prostatic adenocarcinoma with cervical lymphadenopathy: two case reports and review of the literature. Otolaryngol Head Neck Surg 125(4): 431-432.

17. Kimura T, Onozawa M, Miyazaki J, Matsuoka T, Joraku A, et al. (2014) Prognostic impact of young age on stage IV prostate cancer treated with primary androgen deprivation therapy. Int J Urol 21(6): 578583. 